\title{
Endothelial Cells May Have Tissue-Specific Origins
}

\author{
Boström $\mathrm{KI}^{1,2}$, Yao $\mathrm{J}^{1}, \mathrm{Wu} \mathrm{X}^{1}$, and Yao $\mathrm{Y}^{{ }^{1,3}}$ \\ ${ }^{1}$ Division of Cardiology, David Geffen School of Medicine at UCLA, Los Angeles, USA \\ ${ }^{2}$ The Molecular Biology Institute at UCLA, Los Angeles, USA \\ ${ }^{3}$ Jonsson Comprehensive Cancer Center at UCLA, Los Angeles, USA
}

*Corresponding author: Yao Y, M.D., Ph.D., Division of Cardiology, David Geffen School of Medicine at UCLA,Box 951679, Los Angeles, CA 90095-1679, Fax: 310-206-8553, Tel: 310-825-3239, E-mail: yyao@ mednet.ucla.edu

Citation: Boström KI, Yao J, Wu X, Yao Y (2018) Endothelial Cells May Have Tissue-Specific Origins. J Cell Biol Histol 1(1): 104. doi: 10.15744/2638-082X.1.104

Received Date: January 11, 2018 Accepted Date: June 06, 2018 Published Date: June 08, 2018

\begin{abstract}
Endothelial heterogeneity reflects many functions performed by endothelial cells (ECs) in various tissues. However, the origin of this heterogeneity is unclear. Here, we report that tissue-specific ECs in lungs, brain and liver co-expressed the lineage markers of their coordinating tissue-specific cells at very early stages. Specifically, we found that the pulmonary EC population was significantly suppressed after pulmonary epithelial-specific (Nkx2.1-Cre mediated) deletion of fetal liver kinase-1 (Flk1). Together, the results suggest that tissues-specific ECs may originate from the same progenitor cells as tissue-specific cells.
\end{abstract}

Keywords: Endothelial cells; Tissue-specific; Differentiation; Heterogeneity

\section{Introduction}

Vascular endothelial cells (ECs) form the interface between tissue and circulating blood. However, the endothelial functions are not limited to lining vascular structures, but include multiple roles in organogenesis and regulation of biological processes [1]. Although ECs from various locations share common features, tissue-specific ECs display distinct morphological features and specific gene signatures [2-6], and coordinate with organ-specific cells to allow for unique structures and functions [4,5]. In the lungs, pulmonary ECs differentiate in parallel with epithelial cells to form gas exchange units, which supply oxygen and release waste $[7,8]$. In the brain, ECs associate with neurons to develop the semipermeable blood-brain barrier, which protects the neuronal environment and balances the biochemical regulation $[9,10]$. In liver, the connections between ECs are discontinuous and allow the hepatocytes to easily communicate with the bloodstream in order to clear toxins and deliver nutrition [11]. However, the origin of tissue-specific ECs remains unclear, and it is not known whether these ECs differentiate from the same progenitor cells as the corresponding tissue-specific cells. In this study, we found that tissue-specific ECs in lungs, brain and liver share tissuespecific lineage markers at early developmental stages. Pulmonary epithelial-specific deletion of Flk1 as directed by a Nkx2.1-Cre transgene decreases the EC population in the lungs. Together, our data suggest that ECs and tissue-specific cells may derive from the same progenitor cells.

\section{Methods}

\section{Animals}

Wild type, $\mathrm{Nkx} 2.1^{\text {cre }}(\mathrm{Tg}(\mathrm{Nkx} 2.1$-cre $) 2 \mathrm{Sand} / \mathrm{J})$ and Flk1 flox/wt $(\mathrm{Kdrtm} 2 \mathrm{Sato} / \mathrm{J})$ mice on C57BL/6J background were obtained from the Jackson Laboratory. All mice were fed a standard chow diet (Diet 8604, HarlanTeklad Laboratory). The studies were reviewed and approved by Institutional Review Board and conducted in accordance with the animal care guideline set by University of California, Los Angeles. The investigation conformed to the National Research Council, Guide for the Care and Use of Laboratory Animals, Eighth Edition (Washington, DC: The National Academies Press, 2011).

\section{Immunofluorescence}

Immunofluorescence was performed as previously described [12]. We used specific antibodies for Flk1 (Cell Signaling Technology, Cat: 9698), Nkx2.1, CD31, Sox2 and Gata4 (Abcam, Cat: ab76013; ab28364; ab97959; ab84593). The nuclei were stained with 4',6-diamidino-2-phenylindole (DAPI, Sigma-Aldrich). 


\section{Flow Cytometric Analysis}

Fluorescence-activated cell sorting (FACS) was performed as previously described [12]. The cells were stained with fluorescein isothiocyanate (FITC)-, phycoerythrin (PE)-, or Alexa Fluor 488 (AF-488)-conjugated antibodies against Nkx2.1, Flk1, Sox2, Gata4 and VE-cadherin (Abcam). Nonspecific fluorochrome- and isotype-matched IgGs (BD Pharmingen) served as controls.

\section{Statistical analysis}

Two-sided t-test was used to analyze the Data. The analyses were performed using GraphPad Instat ${ }^{\oplus}$, version 3.0 (GraphPad Software). Data represent mean + standard deviation (SD). P-values less than 0.05 were considered significant, and experiments were repeated a minimum of three times. Data were analyzed for statistical significance by ANOVA with post hoc Tukey's analysis.

\section{Results}

\section{Pulmonary endothelial cells with Nkx2.1 expression}

Expression of the transcription factor Nkx2.1 is a hallmark of pulmonary specification $[13,14]$. To investigate the possibility that pulmonary ECs are derived from Nkx2.1-positive progenitor cells at an early stage of lung development, we examined wild type lungs at E10.5 where the pulmonary epithelium first appears after the lung buds emerge from the foregut endoderm [13]. We found ECs that were double-positive for endothelial marker (Flk1 or CD31) and Nkx2.1 adjacent to epithelial cells, which only expressed Nkx2.1 (Figure 1a-b), suggesting that both ECs and epithelial cells derive from Nkx2.1 positive progenitor cells. Flow cytometry results confirmed that a group of ECs in E10.5 lung endoderm co-expressed Nkx2.1 and the endothelial marker VE-cadherin (Figure 1c).
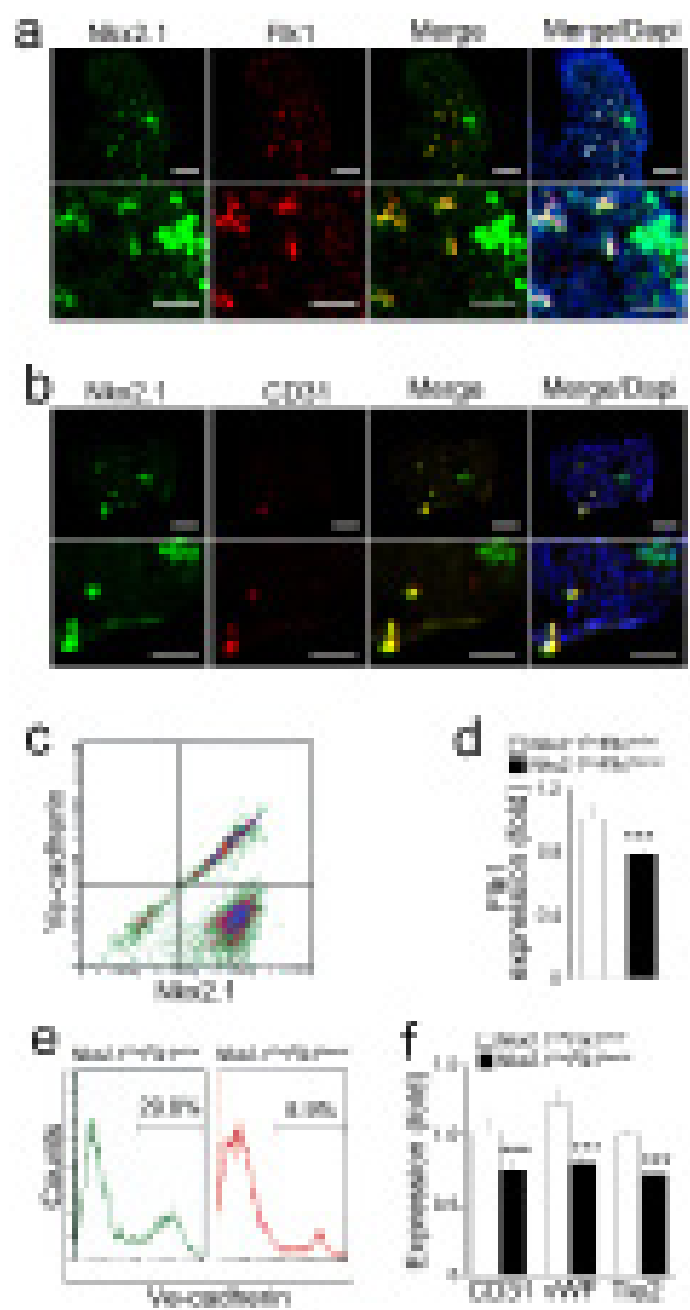

(a-b) Co-expression of Nkx2.1 with Flk1 (a) or CD31 (b) in E10.5 embryonic lungs of wild type mice, as shown by immunostaining. Top: low magnification. Scale bar, $100 \mu \mathrm{m}$. Bottom: high magnification. Scale bar, $50 \mu \mathrm{m}$.

(c) E10.5 embryonic lungs of wild type mice were enzymatically dispersed and analyzed by using flow cytometry.

(d) Expression of Flk1 in E10.5 embryonic lungs of $N k x 2.1^{\text {Cre }} F l k 1^{w t / w t}$ and $N k x 2.1^{\text {Cre }} F l k 1^{f l o x / w t}$ mice.

(e) E10.5 embryonic lungs of $N k x 2.1^{C r e f l k} 1^{w t / w t}$ and $N k x 2.1^{C r e} f l k 1^{f l o x / w t}$ were enzymatically dispersed. The VE-cadherin positive EC population was analyzed by using flow cytometry.

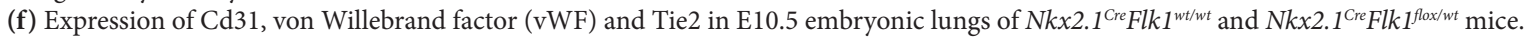

Figure 1: Pulmonary ECs expressing Nkx2.1 in early developmental stages. 
To determine the impact of reducing Flk1 expression in Nkx2.1 positive cells on the pulmonary EC population, we generated $N k x 2.1^{\text {Cre }} F l k 1^{f l o x / w t}$ mice, in which Cre expression is driven by the Nkx2.1 promoter to reduce Flk1 (Figure 1d). We examined the pulmonary EC population of $N k \times 2.1^{\text {Cre }} F l k 1^{f l o x / w t}$ mice by flow cytometric analysis, and found a significant decrease in the EC population (Figure 1e). The real-time PCR also showed a reduction of other endothelial markers, CD31, von Willebrand factor (vWF) and Tie2, in the lungs of $N k x 2.1^{\mathrm{Cre}} f l k 1^{f l o x / w t}$ mice, suggesting that Nkx2.1-positive progenitor cells are the origins of pulmonary ECs.

To further characterize Nkx2.1 positive ECs, we examined the co-expression of Nkx2.1 with Flk1 or Flt1 in wild type embryonic lungs at different time points. The results showed that, from E11.5 to E18.5, the total numbers of Flk1 and Flt1 positive cells increased, but the population of Flk1/Nkx2.1 or Flt1/Nkx2.1 double-positive cells decreased from E14.5 and were barely detectable at E18.5 when the vascular networks formed (Figure 2a-c). The results suggest that pulmonary ECs are derived from the same progenitors as epithelial cells but commit to vascular lineage during the lung development.
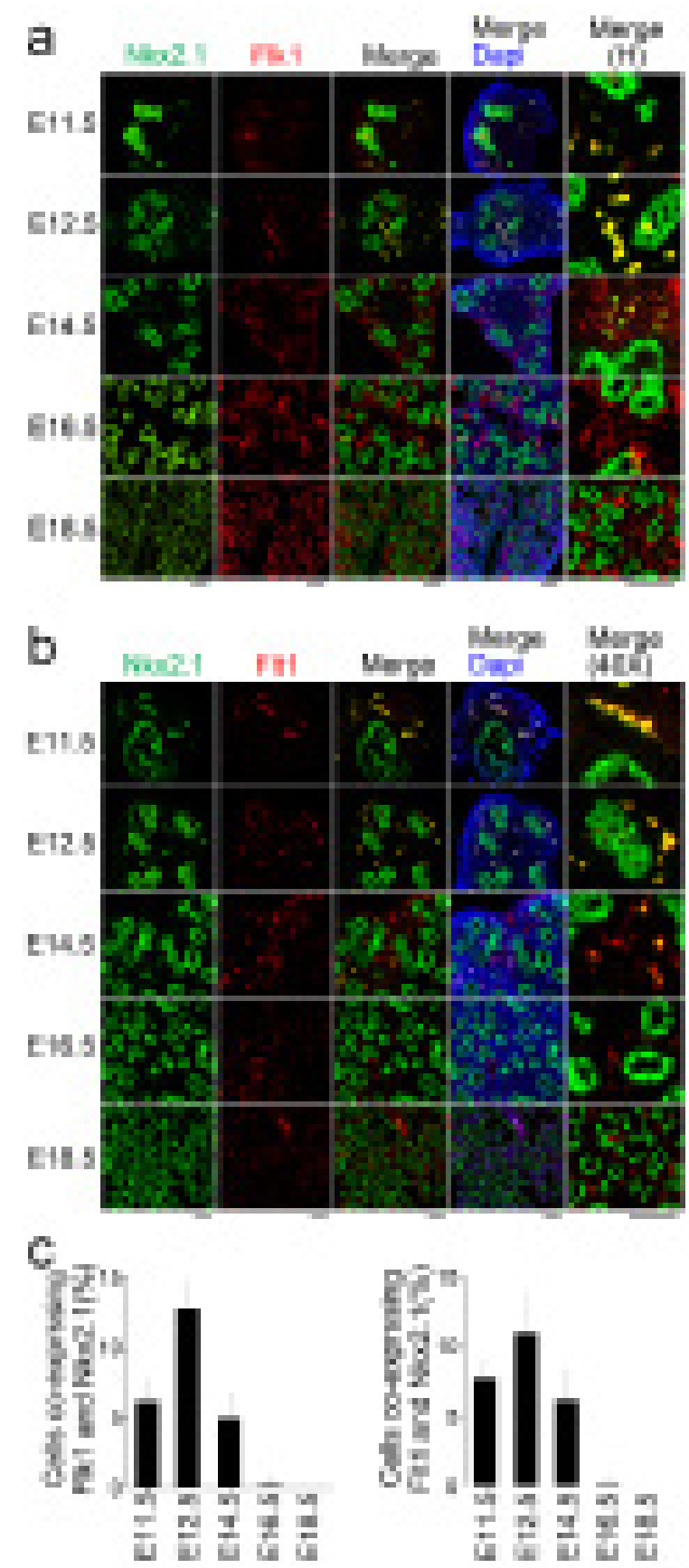

(a-b) Co-expression of Nkx2.1 with Flk1 (a) or Flt1 (b) in embryonic lung from E11.5 to E18.5. Scale bar, $100 \mu \mathrm{m}$.

(c) Percentage of Nkx2.1 and Flk1 or Nkx2.1 and Flt1 double positive cells in embryonic lung from E11.5 to E18.5. Embryos, $n=4$; High-power fields per assessment, $n=5$.

Figure 2: Co-expression of Nkx2.1 with Flk1 and Flt1 in embryonic lung. 


\section{Brain endothelial cells with Sox2 expression}

Sox2 is a key regulator of neuronal differentiation and brain development [15]. To investigate whether brain ECs are derived from Sox2-positive cells, we examined wild type brain at E10.5 and E14. We found Flk1 and Sox2 double positive ECs adjacent to the developing brain cells, which only expressed Sox2 (Figure 3a), suggesting that these ECs and brain cells are derived from the same progenitor cells. We dissected E12.5 embryonic brains and analyzed the cell populations by flow cytometric analysis. We found that a group of ECs co-expressed Sox2 and the endothelial marker VE-cadherin (Figure 3b). However, similar to the lungs, the population of Flk1/Sox2 double-positive cells were undetectable after E18.5 when vascular network formed (Figure 3a).
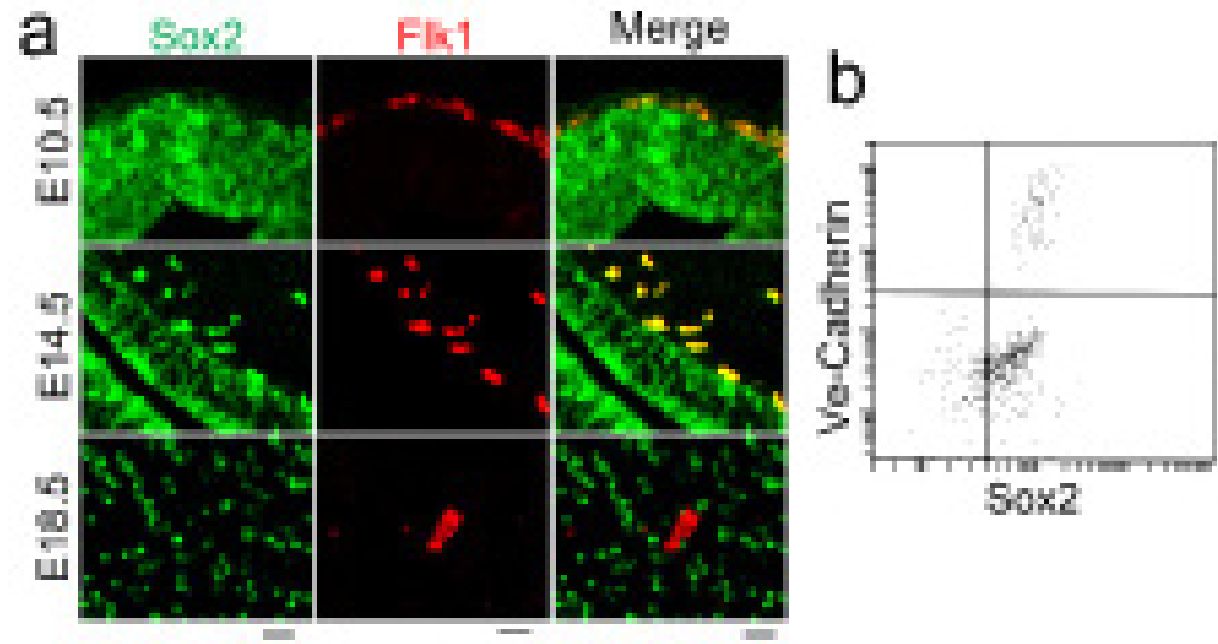

$50 \times 2$

(a) Expression of Sox2 and Flk1 in E10.5 (top), E14.5 (middle) and E18.5 (bottom) embryonic brains of wild type mice, as shown by immunostaining. Scale bar, $50 \mu \mathrm{m}$.

(b) E12.5 embryonic brains of wild type mice were enzymatically dispersed and analyzed by using flow cytometry.

Figure 3: Brain ECs expressing Sox2 at early developmental stages.

\section{Hepatic endothelial cells with Gata4 expression}

Gata4 is an early driver of hepatic differentiation [16]. We examined wild type embryonic liver at E10.5 and E14.5, and found that Flk1/Gata4 double-positive ECs appeared in the tissue surrounded by the hepatocytes, which only expressed Gata4 (Figure 4a). We further dissected E12.5 embryonic livers, and analyzed cell populations by using flow cytometry. We found that the population of VE-cadherin positive ECs co-expressed Gata4, suggesting that both ECs and hepatocytes are derived from Gata4-positive progenitor cells (Figure 4b). Similar to the lungs and brain, after E18.5, the population of Flk1/Gata4 double-positive cells were undetectable in the vascular networks (Figure $4 \mathrm{a}$ ).

Together, our results suggest that early tissue-specific ECs may be derived from the same progenitor cells as their neighboring organ-specific cells.

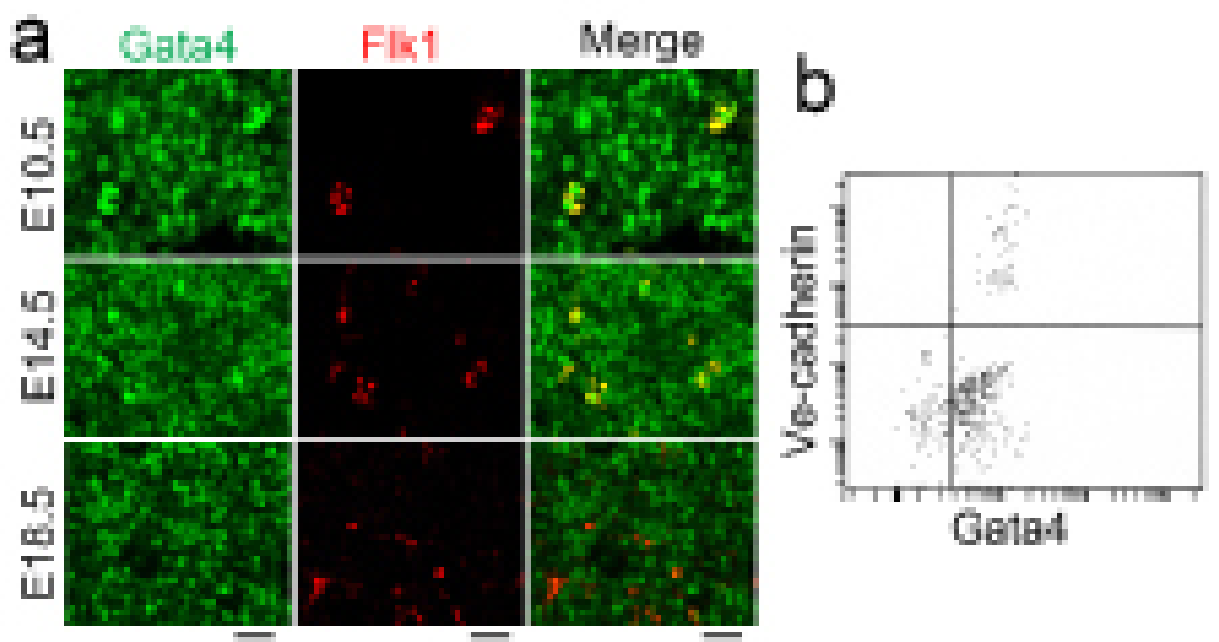

(a) Expression of Gata4 and Flk1 in E10.5 (top), E14.5 (middle) and E18.5 (bottom) embryonic livers of wild type mice, as shown by immunostaining. Scale bar, $50 \mu \mathrm{m}$.

(b) E12.5 embryonic livers of wild type mice were enzymatically dispersed and analyzed by using flow cytometry.

Figure 4: Hepatic ECs expressing Gata4 at early developmental stages. 


\section{Discussion}

In this study, we investigated if tissue-specific ECs are derived from the same progenitor cells as the organ-specific cells. We found that, at early developmental stages, ECs in lungs, brain and liver express tissue-specific markers, which are reported as the important early drivers for the development of these organs [13-16]. When deleting Flk1 expression in pulmonary Nkx2.1 positive cells, we found a decrease in the pulmonary EC population. This is the first study to uncover that the development of tissue-specific ECs may follow the same early path as tissue-specific cells, and may explain the reason for heterogeneity of ECs in different tissues. Furthermore, the same origin of ECs and tissue-specific cells may facilitate the coordination between vascularization and tissue formation during development and tissue repair.

Co-expression of endothelial and tissue-specific markers were detected in ECs at early stages, after which the tissue-specific markers vanished as the vascular networks started to form. This may mark the divergence in developmental direction between ECs and organ-specific cells towards different functions. However, ECs and organ-specific cells may continue to communicate and coordinate the responses to focal and systemic stimuli. Such coordination has been demonstrated in normal development, and may also contribute to the progression of disease.

Our previous studies have shown that ECs are able to transition into other cell lineages through endothelial-mesenchymal transitions [12]. Here, we provide evidence that the endothelial lineage co-exist with tissue-specific lineages during early stages of organogenesis, which support a considerable plasticity in ECs. This plasticity enables ECs to contribute cells to normal development, such as neurogenesis and cardiogenesis [17,18], but also to the progression of pulmonary hypertension [19], atherosclerosis [20], vascular calcification [21], cardiac and renal fibrosis [22,23], fibrodysplasia ossificans progressive [24], and cancer[25].

Expression of brachyury is essential for the development of the primitive streak, which has a role in the development of multiple organs [26]. Lineage tracing has shown that Flk1 positive cell derived from brachyury positive mesodermal cells can differentiate into embryonic ECs [27]. Since deficiency of Flk1 leads to lack of embryonic vessels and lethality [28], the origin of tissue-specific ECs and formation of vascular network is unclear. It was previously hypothesized that brachyury/Flk1 double positive mesodermal cells migrate into the tissues to generate ECs and vascular networks. However, this hypothesis is unable to explain the heterogeneity of ECs in different tissues. Meanwhile, lineage tracing further shows that brachyury/Flk1 double positive cells also differentiate into non-lineage lineages, such as skeletal muscle and cardiomycytes [27]. This suggests the possibility that tissue-specific ECs and organ-specific cells have the same developmental origin. Here, we find the Flk1 positive ECs co-express tissue-specific markers in several organs, which supports that early populations of tissue-specific ECs may have the same origins as organ-specific cells.

\section{Acknowledgments}

Funding for this work was provided in part by NIH grants NS79353, HL30568, HL81397, and HL112839.

\section{References}

1. Coultas L, Chawengsaksophak K, Rossant J (2005) Endothelial cells and VEGF in vascular development. Nature 438(7070): 937-45.

2. Tse D, Stan RV (2010) Morphological heterogeneity of endothelium. Semin Thromb Hemost 36: 236-45.

3. Aird WC (2012) Endothelial cell heterogeneity. Cold Spring Harb Perspect Med 2: a006429.

4. Wilson HK, Canfield SG, Shusta EV, Palecek SP (2014) Concise review: tissue-specific microvascular endothelial cells derived from human pluripotent stem cells. Stem Cells 32: 3037-45.

5. Nolan DJ, Ginsberg M, Israely E, Palikuqi B, Poulos MG, et al. (2013) Molecular signatures of tissue-specific microvascular endothelial cell heterogeneity in organ maintenance and regeneration. Dev Cell 26: 204-19.

6. Augustin HG, Koh GY (2017) Organotypic vasculature: From descriptive heterogeneity to functional pathophysiology. Science 357(6353). pii: eaal2379.

7. Ryan JW, Ryan US (1977) Pulmonary endothelial cells. Fed Proc 36: 2683-91.

8. Rafii S, Butler JM, Ding BS (2016) Angiocrine functions of organ-specific endothelial cells. Nature 529(7586): 316-25.

9. Levin E, Scicli G (1969) Brain barrier phenomena. Brain Res 13: 1-12.

10. Pozhilenkova EA, Lopatina OL, Komleva YK, Salmin VV, Salmina AB (2017) Blood-brain barrier-supported neurogenesis in healthy and diseased brain. Rev Neurosci 28: 397-415.

11. Arias IM (1990) The biology of hepatic endothelial cell fenestrae. Prog Liver Dis 9: 11-26.

12. Yao Y, Jumabay M, Ly A, Radparvar M, Cubberly MR, et al. (2013) A role for the endothelium in vascular calcification. Circ Res 113: 495-504.

13. Herriges M, Morrisey EE (2014) Lung development: orchestrating the generation and regeneration of a complex organ. Development 141: $502-13$.

14. Minoo P, Su G, Drum H, Bringas P, Kimura S (1999) Defects in tracheoesophageal and lung morphogenesis in Nkx2.1(-/-) mouse embryos. Dev Biol 209: 60-71.

15. Amador-Arjona A, Cimadamore F, Huang CT, Wright R, Lewis S, et al. (2015) SOX2 primes the epigenetic landscape in neural precursors enabling proper gene activation during hippocampal neurogenesis. Proc Natl Acad Sci USA 112: E1936-45.

16. Borok MJ, Papaioannou VE, Sussel L (2016) Unique functions of Gata4 in mouse liver induction and heart development. Dev Biol 410: $213-22$.

17. Thiery JP (2003) Epithelial-mesenchymal transitions in development and pathologies. Current opinion in cell biology 15: 740-6.

18. Kalluri R, Neilson EG (2003) Epithelial-mesenchymal transition and its implications for fibrosis. J Clin Invest 112: $1776-84$. 
19. Ranchoux B, Antigny F, Rucker-Martin C, Hautefort A, Pechoux C, et al. (2015) Endothelial-to-mesenchymal transition in pulmonary hypertension. Circulation 131: 1006-18.

20. Chen PY, Qin L, Baeyens N, Li G, Afolabi T, et al. (2015) Endothelial-to-mesenchymal transition drives atherosclerosis progression. J Clin Invest 125: 4514-28.

21. Yao J, Guihard PJ, Blazquez-Medela AM, Guo Y, Moon JH, et al. (2015) Serine Protease Activation Essential for Endothelial-Mesenchymal Transition in Vascular Calcification. Circ Res 117: 758-69.

22. Kovacic JC, Mercader N, Torres M, Boehm M, Fuster V (2012) Epithelial-to-mesenchymal and endothelial-to-mesenchymal transition: from cardiovascular development to disease. Circulation 125: 1795-808.

23. Kriz W, Kaissling B, Le Hir M (2011) Epithelial-mesenchymal transition (EMT) in kidney fibrosis: fact or fantasy? J Clin Invest 121: 468-74.

24. Medici D, Shore EM, Lounev VY, Kaplan FS, Kalluri R, et al. (2010) Conversion of vascular endothelial cells into multipotent stem-like cells. Nat Med 16: 1400-6.

25. Gonzalez DM, Medici D (2014) Signaling mechanisms of the epithelial-mesenchymal transition. Science signaling. 7: re8.

26. Wilkinson DG, Bhatt S, Herrmann BG (1990) Expression pattern of the mouse T gene and its role in mesoderm formation. Nature 343: 657-9.

27. Yamaguchi TP, Dumont DJ, Conlon RA, Breitman ML, Rossant J (1993) flk-1, an flt-related receptor tyrosine kinase is an early marker for endothelial cell precursors. Development 118: 489-98.

28. Shalaby F, Ho J, Stanford WL, Fischer KD, Schuh AC, et al. (1997) A requirement for Flk1 in primitive and definitive hematopoiesis and vasculogenesis. Cell 89: 981-90.

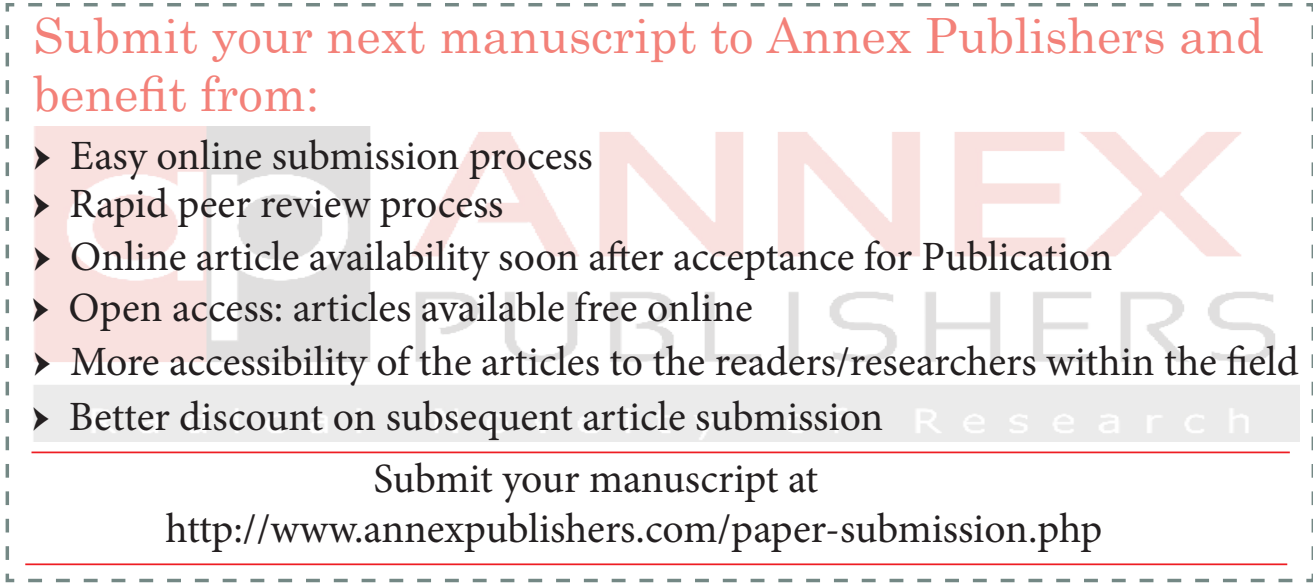

